\title{
Light-induced bound electron states in two-dimensional systems: Contribution to electron transport
}

\author{
O. V. Kibis * M. V. Boev, and V. M. Kovalev \\ Department of Applied and Theoretical Physics, Novosibirsk State Technical University, \\ Karl Marx Avenue 20, Novosibirsk 630073, Russia
}

\begin{abstract}
In two-dimensional (2D) electron systems, an off-resonant high-frequency circularly polarized electromagnetic field can induce the quasi-stationary bound electron states of repulsive scatterers. As a consequence, the resonant scattering of conduction electrons through the quasi-stationary states and the capture of conduction electrons by the states appear. The present theory describes the transport properties of $2 \mathrm{D}$ electron gas irradiated by a circularly polarized light, which are modified by these processes. Particularly, it is demonstrated that irradiation of $2 \mathrm{D}$ electron systems by the off-resonant field results in the quantum correction to conductivity of resonant kind.
\end{abstract}

\section{INTRODUCTION}

The control of physical properties of various quantum systems by an off-resonant high-frequency electromagnetic field became the important and established research area which resulted in many fundamental effects (see, e.g., Refs. 1 9). Since the frequency of the field lies far from characteristic resonant frequencies of an irradiated system (off-resonant field), the field can not be absorbed by electrons and only interacts non-resonantly with them ("dresses" them). As a result, the behavior of dressed electrons varies as a function of the dressing field. To clarify the field-induced features of electronic properties of low-dimensional systems, many works dedicated to the electromagnetic dressing of various nanostructures - including quantum rings ${ }^{10}-13$, quantum wells ${ }^{14}-18$, topological insulators ${ }^{19}-28$, graphene and related 2D materials ${ }^{29}-43$, etc - were published.

Among variety of phenomena in periodically driven systems, the effect of dynamical stabilization should be noted especially. Generally, the dynamical stabilization is the fundamental physical effect which consists in the stabilization of initially unstable systems by oscillating external actions (see, e.g., Ref. 2). Phenomenologically, this effect was known for a long time to any circus juggler who hold various objects in balance by vibrational movements of its hands. First correct description of the effect in the frame of classical Hamilton mechanics was done by P. L. Kapitza who suggested the simple mechanical model based on a pendulum 44 . The Kapitza pendulum is the system consisting of a point mass attached to a light inextensible rod connected to a vibrating suspension. In the case of fixed point of suspension, this model describes the conventional mathematical pendulum for which there are two points of equilibrium (the lower and the upper). The equilibrium of the pendulum in the upper point is unstable and any infinitesimal perturbation leads to loss of the equilibrium. However, in contravention of intuition, the upper (vertical) position of the pendulum can be steady in the case of fast oscillating suspension. Thus, the Kapitza pendulum acquires the quasi-stable equilibrium (the local minimum of its potential energy) at the upper point which corresponds to the maximum of its potential energy in the absence of oscillations. This effect is of universal nature and occurs in many different areas of physics. For example, an oscillating laser field can lead to stabilization of charged ionic systems whose components repulse each other according to the Coulomb law and, therefore, cannot exist as a whole without the field ${ }^{45}$. Despite the long prehistory of the effect of dynamical stabilization, its possible manifestations in nanostructures still wait for detailed study. To fill partially this gap in the theory, we analyzed recently the behavior of various repulsive potentials in nanostructures driven by an oscillating field in context of the dynamical stabilization ${ }^{46}$. Particularly, it was found that a circularly polarized electromagnetic field can induce the local minima of potential energy in the core of $2 \mathrm{D}$ repulsive potentials. As a consequence, the quasi-stationary electron states confined near the local minima appear. The present article is dedicated to theoretical analysis of the electron transport in $2 \mathrm{D}$ systems modified by the light-induced quasi-stationary electron states bound at repulsive scatterers.

The article is organized as follows. In Sec. II, we develop the theory of the light-induced quasi-stationary electron states bound at short-range scatterers modeled by the repulsive delta-potential. In Sec. III, the Boltzmann kinetic equation taking into account the scattering and capture of conduction electrons by the quasistationary bound states is solved. In Sec. IV, analysis of the conductivity of the irradiated $2 \mathrm{D}$ electron system is performed. The last two sections of the article contain conclusion and acknowledgments.

\section{MODEL}

Let us consider a 2D electron system in the $(x, y)$ plane irradiated by a circularly polarized electromagnetic wave propagating along the $z$ axis with the vector potential $\mathbf{A}(t)=\left(A_{x}, A_{y}\right)=\left[c E_{0} / \omega\right](\sin \omega t, \cos \omega t)$, where $E_{0}$ is the electric field amplitude of the wave, and $\omega$ is the wave frequency which lies far from characteristic resonant frequencies of the electron system (the off-resonant dressing field) [see Fig. 1a]. The physical properties of 
the dressed 2D electron system in the presence of a scatterer with the repulsive potential $U(\mathbf{r})$ are described by the Hamiltonian

$$
\hat{\mathcal{H}}=\frac{[\hat{\mathbf{p}}-e \mathbf{A}(t) / c]^{2}}{2 m_{e}}+U(\mathbf{r}),
$$

where $\hat{\mathbf{p}}=\left(\hat{p}_{x}, \hat{p}_{y}\right)$ is the plane momentum operator, $m_{e}$ is the effective electron mass, $e$ is the electron charge, and $\mathbf{r}=(x, y)$ is the plane radius vector of an electron. Applying the Kramers-Henneberger unitary transformation 47.48 ,

$$
\hat{\mathcal{U}}(t)=\exp \left\{\frac{i}{\hbar} \int_{-\infty}^{t}\left[\frac{e}{m_{e} c} \mathbf{A}\left(t^{\prime}\right) \hat{\mathbf{p}}-\frac{e^{2}}{2 m_{e} c^{2}} A^{2}\left(t^{\prime}\right)\right] d t^{\prime}\right\},
$$

the transformed Hamiltonian (11) reads

$$
\begin{aligned}
\hat{\mathcal{H}}^{\prime} & =\hat{\mathcal{U}}^{\dagger}(t) \hat{\mathcal{H}} \hat{\mathcal{U}}(t)-i \hbar \hat{\mathcal{U}}^{\dagger}(t) \partial_{t} \hat{\mathcal{U}}(t) \\
& =\frac{\hat{\mathbf{p}}^{2}}{2 m_{e}}+U\left(\mathbf{r}-\mathbf{r}_{0}(t)\right),
\end{aligned}
$$

where the radius vector $\mathbf{r}_{0}(t)=\left(-r_{0} \cos \omega t, r_{0} \sin \omega t\right)$ describes the classical circular trajectory of electron movement in the circularly polarized field, and

$$
r_{0}=\frac{|e| E_{0}}{m_{e} \omega^{2}}
$$

is the radius of the trajectory $\underline{49}$. Physically, the unitary transformation (2) corresponds to the transition from the laboratory reference frame to the rotating reference frame,

$$
\mathbf{r} \rightarrow \mathbf{r}-\mathbf{r}_{0}(t)
$$

Expanding the oscillating potential in Eq. (3) into a Fourier series,

$$
U\left(\mathbf{r}-\mathbf{r}_{0}(t)\right)=U_{0}(\mathbf{r})+\left[\sum_{n=1}^{\infty} U_{n}(\mathbf{r}) e^{i n \omega t}+\text { c. c. }\right],
$$

the transformed Hamiltonian (3) can be rewritten as

$$
\hat{\mathcal{H}}^{\prime}=\frac{\hat{\mathbf{p}}^{2}}{2 m_{e}}+U_{0}(\mathbf{r})+\left[\sum_{n=1}^{\infty} U_{n}(\mathbf{r}) e^{i n \omega t}+\text { c. c. }\right],
$$

where

$$
U_{0}(\mathbf{r})=\frac{1}{2 \pi} \int_{-\pi}^{\pi} U\left(\mathbf{r}-\mathbf{r}_{0}(t)\right) d(\omega t)
$$

is the stationary part of the potential, which is responsible for the smooth motion of $2 \mathrm{D}$ electrons, and $U_{n}(\mathbf{r})$ are the Fourier coefficients of the oscillating potential. The stationary potential (8) should be treated as a repulsive potential dressed by an oscillating field (dressed potential). The specific feature of the dressed potential (8) is the local minimum existing near $r=0$ if the field is strong enough $\frac{46}{}$ (see Fig. 1b). As a consequence of the (a)
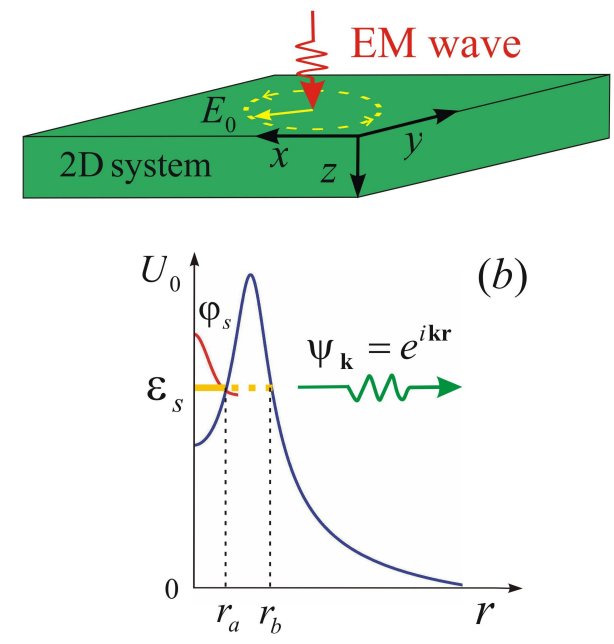

FIG. 1: Sketch of the system under consideration: (a) 2D electron system irradiated by a circularly polarized electromagnetic wave with the electric field amplitude $E_{0}$; (b) The $2 \mathrm{D}$ scattering repulsive potential dressed by a circularly polarized field, $U_{0}(r)$, which has the local minimum at $r=0$ and contains the bound electron state with the wave function $\varphi_{s}$ (the Gaussian-like red line) and the energy $\varepsilon_{s}$ (the horizontal yellow line). The bound state is quasi-stationary and can decay due to the tunneling through the potential barrier between the two turning points, $r_{a}$ and $r_{b}$. As a result of the tunneling, the emission of free electron with the wave function $\psi_{\mathbf{k}}=e^{i \mathbf{k r}}$ (the green wave arrow) from the bound state takes place.

local minimum, the domain of attraction takes place in the core of the repulsive potential. This attraction results in confinement of an electron at the repulsive potential and the bound electron state with the energy $\varepsilon_{s}$ and the localized wave function $\varphi_{s}$ appears. In the following, we will assume that the field frequency, $\omega$, satisfies the two conditions: Firstly,

$$
\omega \tau_{e} \gg 1
$$

where $\tau_{e}$ is the mean free time of conduction electrons; secondly, the field frequency lies far from resonant frequencies of the bound state. Under the first condition, scattering processes cannot destroy the bound state, whereas the second condition allows to neglect the effect of the oscillating terms of the Hamiltonian (7) on the bound state. As a consequence, the electron dynamics of an electron confined at a scatterer can be described solely by the stationary part of the Hamiltonian,, 4

$$
\hat{\mathcal{H}}_{0}=\frac{\hat{\mathbf{p}}^{2}}{2 m_{e}}+U_{0}(\mathbf{r}) .
$$

The field-induced local minimum of the dressed potential $U_{0}(\mathbf{r})$ forms the quantum well which separates the bound electron state inside the well, $\varphi_{s}$, from the states of free conduction electrons outside the well, $\psi_{\mathbf{k}}=e^{i \mathbf{k r}}$, 
by the potential barrier between the turning points, $r_{a}$ and $r_{b}$ (see Fig. 1b). Physically, the electron tunneling through the barrier, $\varphi_{s} \rightarrow \psi_{\mathbf{k}}$, results in finite lifetime of the bound state and the broadening of its energy. To find the bound state energy $\varepsilon$ and the energy broadening $\Gamma$, we have to solve the Schrödinger equation, $\hat{\mathcal{H}}_{0} \psi=\varepsilon \psi$, with the Hamiltonian (10). Let us model the repulsive potential $U(\mathbf{r})$ with the delta function,

$$
U(\mathbf{r})=u_{0} \delta(\mathbf{r})
$$

where $u_{0}>0$ is the strength of the repulsive potential. Substituting the potential (11) into Eq. (8), we arrive at the dressed delta-potential,

$$
U_{0}(\mathbf{r})=\frac{u_{0} \delta\left(r-r_{0}\right)}{2 \pi r_{0}} .
$$

Thus, the circularly polarized field turns the repulsive delta potential (11) into the delta potential barrier of ring shape (12). As a consequence, quasi-stationary electron states confined inside the area fenced by the ring-shape barrier $\left(0<r<r_{0}\right)$ can exist. Substituting the dressed potential (12) into Eq. (10), we arrive at the Hamiltonian

$$
\hat{\mathcal{H}}_{0}=-\frac{\hbar^{2}}{2 m_{e} r}\left[\frac{\partial}{\partial r}\left(r \frac{\partial}{\partial r}\right)+\frac{1}{r} \frac{\partial^{2}}{\partial \varphi^{2}}\right]+\frac{u_{0} \delta\left(r-r_{0}\right)}{2 \pi r_{0}},
$$

where $\varphi$ is the azimuth angle in the 2D plane. The eigenfunction of the Hamiltonian, $\psi$, which corresponds to an electron confined in the area $0<r<r_{0}$, must be finite at $r=0$ and satisfy the condition $\left.\psi\right|_{r \rightarrow \infty} \propto e^{i k r}$, where $k=\sqrt{2 m_{e} \varepsilon} / \hbar$ is the electron wave vector. Solving the Schrödinger problem with the Hamiltonian (13) under these conditions and assuming $\alpha=2 \hbar^{2} / m_{e} u_{0} \ll 1$ (see Appendix A), we arrive at the sought energy spectrum of an electron confined inside the ring-shape delta-barrier, $\varepsilon=\varepsilon_{n m}-i \Gamma_{n m} / 2$, and the corresponding wave functions, $\psi_{n m}$, where the energy of quasi-discrete electron level is

$$
\varepsilon_{n m}=\frac{\hbar^{2} \bar{z}_{n m}^{2}}{2 m_{e} r_{0}^{2}}+\mathcal{O}(\alpha)
$$

the broadening of the energy level is

$$
\Gamma_{n m}=\frac{4 \varepsilon_{n m} \alpha^{2}}{N_{m}^{3}\left(\bar{z}_{n m}\right)\left[J_{m+1}\left(\bar{z}_{n m}\right)-J_{m-1}\left(\bar{z}_{n m}\right)\right]}+\mathcal{O}\left(\alpha^{3}\right),
$$

the wave functions are

$$
\begin{aligned}
\psi_{n m} & =\frac{e^{i m \varphi}}{\sqrt{\pi} r_{0} J_{m+1}\left(\bar{z}_{n m}\right)}\left\{\begin{aligned}
J_{m}\left(\frac{\bar{z}_{n m} r}{r_{0}}\right), & 0<r \leq r_{0} \\
0, & r \geq r_{0}
\end{aligned}\right. \\
& +\mathcal{O}(\alpha),
\end{aligned}
$$

$J_{m}(\xi)$ is the $m$ th Bessel function of the first kind, $N_{m}(\xi)$ is the $m$ th Bessel function of the second kind (the Neumann function), $\bar{z}_{n m}$ is the $n$th zero of the $m$ th Bessel function of the first kind (i.e. $J_{m}\left(\bar{z}_{n m}\right)=0$ ), and $n=1,2,3, \ldots$ is the principal quantum number. As expected, in the limiting case of $u_{0} \rightarrow \infty$, the energy broadening (15) is zero and the quasi-stationary electron state (14) turns into the stationary one.

Generally, the discussed bound state exists if its energy broadening is small as compared with the characteristic distance between the neighbor energy levels. Therefore, we will restrict the following consideration by the ground bound state, $\varphi_{s}(r)=\psi_{10}(r)$, which has the minimal broadening $\Gamma_{s}=\Gamma_{10}$. It follows from Eqs. (14)-(15) that the energy of the ground bound state and its broadening read

$$
\begin{gathered}
\varepsilon_{s}=\frac{\hbar^{2} \bar{z}_{10}^{2}}{2 m_{e} r_{0}^{2}}, \\
\Gamma_{s}=\frac{2 \varepsilon_{s} \alpha^{2}}{N_{0}^{3}\left(\bar{z}_{10}\right) J_{1}\left(\bar{z}_{10}\right)},
\end{gathered}
$$

where $\bar{z}_{10} \approx 2.4$ is the first zero of the zeroth Bessel function of the first kind. Correspondingly, the applicability condition of Eqs. (17)-(18) can be written as

$$
\frac{\Gamma_{s}}{\varepsilon_{s}} \ll 1 .
$$

The condition (19) can be satisfied if the scattering potential (11) is strong enough, i.e. $\alpha=2 \hbar^{2} / m_{e} u_{0} \ll 1$. It should be noted that this condition does not depend on the strength of the alternating field because of the delta-function singularity of the model repulsive potential (11). If the repulsive potential $U(\mathbf{r})$ is described by any smooth function, the quasi-stationary state - or, what is the same, the field-induced local minimum of potential energy in the core of the repulsive potential takes place if the alternating field is strong enough $\underline{46}$.

\section{KINETIC EQUATION}

The field-induced modification of a repulsive potential discussed above results in both capture of conduction electrons at the bound state and to scattering them through the quasi-discrete energy level $\varepsilon_{s}$. Let us analyze the effect of these processes on electron transport under the stationary field $\mathbf{E}$ applied to the $2 \mathrm{D}$ system of the area $S$. In the laboratory reference frame, the conventional Boltzmann kinetic equation (see, e.g., Ref. 50) can be written as

$$
\left.\left.\frac{\partial f_{\mathbf{k}}}{\partial t}\right]_{\text {field }}+\frac{\partial f_{\mathbf{k}}}{\partial t}\right]_{\text {scatter }}=0
$$

where $f_{\mathbf{k}}$ is the distribution function of conduction electrons with the wave vector $\mathbf{k}$,

$$
\left.\frac{\partial f_{\mathbf{k}}}{\partial t}\right]_{\text {field }}=-\frac{\partial f_{\mathbf{k}}}{\partial \mathbf{k}} \frac{e \mathbf{E}}{\hbar}
$$


is the field term describing the transport of conduction electrons under the weak stationary electric field $\mathbf{E}$,

$$
\left.\frac{\partial f_{\mathbf{k}}}{\partial t}\right]_{\text {scatter }}=\sum_{\mathbf{k}^{\prime}}\left[f_{\mathbf{k}^{\prime}}\left(1-f_{\mathbf{k}}\right)-f_{\mathbf{k}}\left(1-f_{\mathbf{k}^{\prime}}\right)\right] w_{\mathbf{k k}^{\prime}}
$$

is the term describing the scattering of conduction electrons, and $w_{\mathbf{k k}^{\prime}}$ is the probability of electron scattering between the states $\mathbf{k}^{\prime}$ and $\mathbf{k}$ per unit time. In the following, we will assume that the light-induced bound states of scatterers can capture only one electron per scatterer because of the Coulomb repulsion between electrons. Then the conservation law for the total number of electrons in the $2 \mathrm{D}$ system reads

$$
N_{e}+N_{s} f_{s}=N_{0}
$$

where $N_{0}$ is the total number of conduction electrons in the $2 \mathrm{D}$ system in the absence of irradiation,

$$
N_{e}=2 \sum_{\mathbf{k}} f_{\mathbf{k}}
$$

is the total number of conduction electrons in the irradiated $2 \mathrm{D}$ system, and $N_{s} f_{s}$ is the total number of electrons captured by scatterers. Correspondingly, $N_{s}$ is the total number of scatterers in the $2 \mathrm{D}$ system, and $f_{s}$ is the distribution function of conduction electrons captured by the scatterers. In Eq. (24) and what follows, the sum symbol $\sum_{\mathbf{k}}$ denotes the summation over all electron states with different wave vectors $\mathbf{k}$, excluding the summation over the spin freedom degrees [see Eq. (B2) in Appendix B].

The distribution function of captured electrons, $f_{s}$, is defined by the balance equation for them,

$$
\sum_{\mathbf{k}} W_{\mathbf{k} s} f_{s}\left(1-f_{\mathbf{k}}\right)=\sum_{\mathbf{k}} W_{s \mathbf{k}} f_{\mathbf{k}}\left(1-f_{s}\right),
$$

where

$$
W_{s \mathbf{k}}=W_{\mathbf{k} s}=\frac{\hbar^{2} \Gamma_{s}}{S m_{e}\left[\left(\varepsilon_{k}-\varepsilon_{s}\right)^{2}+\left(\Gamma_{s} / 2\right)^{2}\right]}
$$

is the probability of capture of a conduction electron with the wave vector $\mathbf{k}$ and the energy $\varepsilon_{k}=\hbar^{2} k^{2} / 2 m_{e}$ by a scatterer, which is derived in Appendix B. Physically, the balance equation (25) means that the probability of electron transition from the bound state to the continuum of free conduction electrons is equal to the probability of the inverse process for any stationary distribution of electrons. Substituting Eq. (26) into Eq. (25) and keeping in mind Eqs. (B2) and (19), the distribution function of conduction electrons captured by scatterers reads

$$
f_{s}=\sum_{\mathbf{k}} W_{s \mathbf{k}} f_{\mathbf{k}}
$$

Since an oscillating field driving $2 \mathrm{D}$ electrons is assumed to satisfy the high-frequency condition (9), one can neglect the collisional absorption of the field by conduction electrons. Therefore, in the absence of the stationary field, $\mathbf{E}=0$, the electronic system is in the thermodynamic equilibrium and the distribution function is $f_{\mathbf{k}}=f^{(0)}\left(\varepsilon_{k}\right)$, where

$$
f^{(0)}\left(\varepsilon_{k}\right)=\frac{1}{\exp \left[\left(\varepsilon_{k}-\varepsilon_{F}\right) / T\right]+1}
$$

is the Fermi-Dirac distribution function, $\varepsilon_{F}$ is the Fermi energy, and $T$ is the temperature. Substituting the distribution function $f_{\mathbf{k}}=f^{(0)}\left(\varepsilon_{k}\right)$ and the probability (26) into Eq. (27), we obtain the equilibrium distribution function of conduction electrons captured by the scatterers,

$$
f_{s}^{(0)}=\frac{1}{\pi} \int_{0}^{\infty} \frac{\left(\Gamma_{s} / 2\right) f^{(0)}\left(\varepsilon_{k}\right) d \varepsilon_{k}}{\left(\varepsilon_{k}-\varepsilon_{s}\right)^{2}+\left(\Gamma_{s} / 2\right)^{2}} .
$$

Certainly, in the limiting case of stationary bound electron state $\left(\Gamma_{s} \rightarrow 0\right)$, the distribution function (28) turns into the Fermi-Dirac function, $f_{s}^{(0)}=f^{(0)}\left(\varepsilon_{s}\right)$. Substituting the equilibrium distribution functions $f_{\mathbf{k}}=f^{(0)}\left(\varepsilon_{k}\right)$ and $f_{s}=f_{s}^{(0)}$ into Eq. (23), we arrive at the equation defining the Fermi energy $\varepsilon_{F}$,

$$
n_{s} f_{s}^{(0)}+n_{e}=n_{0}
$$

where $n_{s}=N_{s} / S$ is the density of scatterers, $n_{0}=N_{e} / S$ is the density of conduction electrons in the absence of irradiation, and

$$
n_{e}=\frac{2}{S} \sum_{\mathbf{k}} f^{(0)}\left(\varepsilon_{k}\right)=-\frac{m_{e} T}{\pi \hbar^{2}} \ln \left[\frac{1}{1+e^{\varepsilon_{F} / T}}\right]
$$

is the density of conduction electrons in the irradiated $2 \mathrm{D}$ system.

Assuming the thermodynamic equilibrium to be weakly perturbed by the stationary field $\mathbf{E}$, the sought distribution functions can be written as $f_{\mathbf{k}}=f^{(0)}\left(\varepsilon_{k}\right)+$ $\Delta f_{\mathbf{k}}$ and $f_{s}=f_{s}^{(0)}+\Delta f_{s}$, where $\Delta f_{\mathbf{k}}$ and $\Delta f_{s}$ are small nonequilibrium additions arisen from the field $\mathbf{E}$. To find these additions, let us assume that the temperature is around zero in order to neglect the phonon scattering of conduction electrons. Then the scattering probability per unit time, $w_{\mathbf{k k}^{\prime}}$, can be written in the explicit form as

$$
w_{\mathbf{k k}^{\prime}}=\left(1-f_{s}\right) w_{\mathbf{k} \mathbf{k}^{\prime}}^{(1)}+f_{s} w_{\mathbf{k k}^{\prime}}^{(2)}
$$

where the first term,

$$
\begin{aligned}
w_{\mathbf{k k}^{\prime}}^{(1)} & =\frac{2 \pi n_{s}}{S \hbar} \mid \gamma_{\mathbf{k k}^{\prime}} U_{\mathbf{k k}^{\prime}} \\
& +\left.\frac{\hbar^{2} \Gamma_{s}}{m_{e}\left(\varepsilon_{k}-\varepsilon_{s}+i \Gamma_{s} / 2\right)}\right|^{2} \delta\left(\varepsilon_{k^{\prime}}-\varepsilon_{k}\right),
\end{aligned}
$$


describes the scattering of conduction electrons by empty scatterers, and the second term,

$$
\begin{aligned}
w_{\mathbf{k k}^{\prime}}^{(2)} & =\frac{2 \pi n_{s}}{S \hbar} \mid \gamma_{\mathbf{k k}^{\prime}} U_{\mathbf{k k}^{\prime}}+u_{\mathbf{k k}^{\prime}} \\
& -\left.\frac{\hbar^{2} \Gamma_{s}}{m_{e}\left(\varepsilon_{k}-\varepsilon_{s}+i \Gamma_{s} / 2\right)}\right|^{2} \delta\left(\varepsilon_{k^{\prime}}-\varepsilon_{k}\right),
\end{aligned}
$$

describes the scattering of conduction electrons by scatterers containing captured electrons. Here $U_{\mathbf{k k}^{\prime}}$ is the matrix element of the Born scattering by the initial potential $U(\mathbf{r}), u_{\mathbf{k k}^{\prime}}$ is the matrix element of the Born scattering by the potential addition $u(\mathbf{r})$ produced by a captured electron, and $\gamma_{\mathbf{k k}^{\prime}}=J_{0}\left[2 k r_{0} \sin (\theta / 2)\right]$ [see Appendix $\mathrm{B}$ for details of derivation of the probabilities (32) and (33)].

The first terms under modulus in Eqs. (32)- 33) describes the conventional potential scattering, whereas the last therm corresponds to the electron scattering through the quasi-discrete energy level $\varepsilon_{s}$ and is physically identical to the Breit-Wigner equation for the resonant scattering 51 . The probabilities (32)- (33) take into account the quantum interference of these two scattering processes, which can manifest itself in electron transport as features of the Fano kind ${ }^{52}$. However, the interference term is small under the condition of small broadening, $\Gamma_{s}$, and, therefore, can be neglected at a first approximation. It should be noted also that the second terms under modulus in Eqs. (32)- (33) are of different signs. Physically, this originates from different intermediate states involved in the resonant scattering described by Eqs. (32) and (33): In the case of an empty scatterer the intermediate state corresponds to the scatterer containing a captured electron, whereas the intermediate state of a scatterer containing a captured electron corresponds to the empty scatterer.

Since the probabilities (32) and (33) describe the elastic scattering of conduction electrons with the isotropic energy spectrum $\varepsilon_{k}=\hbar^{2} k^{2} / 2 m_{e}$ and the scattering potentials $U(\mathbf{r})$ and $u(\mathbf{r})$ are assumed to be axially symmetric in the $2 \mathrm{D}$ plane, the total probability (31) can be easily rewritten as a function of the angle $\theta=\widehat{\mathbf{k}, \mathbf{k}^{\prime}}$ and the modulus of electron wave vector $k$. Therefore, it is convenient to write the scattering matric elements as functions of these arguments, $U_{\mathbf{k k}^{\prime}}=U_{k}(\theta)$ and $u_{\mathbf{k k}^{\prime}}=u_{k}(\theta)$. Then the relaxation time approximation is applicable to solve the Boltzmann kinetic equation (20) and the sought nonequilibrium distribution functions read

$$
\begin{aligned}
\Delta f_{\mathbf{k}} & =\left[-\frac{\partial f^{(0)}\left(\varepsilon_{k}\right)}{\partial \varepsilon_{k}}\right] e \tau_{k} \mathbf{v}_{\mathbf{k}} \mathbf{E}, \\
\Delta f_{s} & =0
\end{aligned}
$$

where $\mathbf{v}_{\mathbf{k}}=\partial_{\mathbf{k}} \varepsilon_{\mathbf{k}} / \hbar$ is the velocity of conduction electron with the wave vector $\mathbf{k}$, the transport relaxation time, $\tau_{k}$, is defined by the expression

$$
\frac{1}{\tau_{k}}=\int_{-\pi}^{\pi}(1-\cos \theta) w_{k}(\theta) d \theta
$$

where the effective scattering probability per unit time, $w_{k}(\theta)$, reads

$$
\begin{aligned}
w_{k}(\theta) & =\frac{n_{s} m_{e}}{2 \pi \hbar^{3}}\left[\left(1-f_{s}\right)\left|J_{0}\left[2 k r_{0} \sin (\theta / 2)\right] U_{k}(\theta)+\frac{\hbar^{2} \Gamma_{s}}{m_{e}\left(\varepsilon_{k}-\varepsilon_{s}+i \Gamma_{s} / 2\right)}\right|^{2}\right. \\
& +f_{s} \mid J_{0}\left[2 k r_{0} \sin (\theta / 2) U_{k}(\theta)+u_{k}(\theta)-\left.\frac{\hbar^{2} \Gamma_{s}}{m_{e}\left(\varepsilon_{k}-\varepsilon_{s}+i \Gamma_{s} / 2\right)}\right|^{2}\right] .
\end{aligned}
$$

Certainly, the distribution functions (34)-(35) satisfy the Boltzmann kinetic equation (20) and can be easily verified by direct substitution into this. Substituting Eqs. (36)-37) into Eq. (34) and summating it over all electronic states, we arrive at the conductivity of the $2 \mathrm{D}$ system

$$
\sigma=\frac{e^{2} m_{e}}{2 \pi \hbar^{2}} \int_{0}^{\infty}\left[-\frac{\partial f^{(0)}\left(\varepsilon_{k}\right)}{\partial \varepsilon_{k}}\right] v_{k}^{2} \tau_{k} d \varepsilon_{k}
$$

\section{RESULTS AND DISCUSSION}

For definiteness, let us restrict the analysis of electron transport by the case of zero temperature, $T=0$, when the conductivity (38) reads

$$
\sigma=\frac{e^{2} n_{e} \tau_{F}}{m_{e}}
$$

where $\tau_{F}$ is the relaxation time (36) for $\varepsilon_{k}=\varepsilon_{F}$. Taking into account Eqs. (28)-(29) and (19), the density of conduction electrons, $n_{e}$, is defined by the equation

$$
n_{e}=n_{0}-\frac{n_{s}}{2}-\frac{n_{s}}{\pi} \tan ^{-1}\left(\frac{2\left[\varepsilon_{F}-\varepsilon_{s}\right]}{\Gamma_{s}}\right),
$$


where $\varepsilon_{F}=\pi \hbar^{2} n_{e} / m_{e}$ is the Fermi energy. In what follows, we will be to consider the physically relevant case of small density of scatterers, $n_{s} \ll n_{0}$. Then the solution of Eq. (40) reads

$$
n_{e}=n_{0}-\frac{n_{s}}{2}-\frac{n_{s}}{\pi} \tan ^{-1}\left(\frac{2\left[\varepsilon_{F 0}-\varepsilon_{s}\right]}{\Gamma_{s}}\right),
$$

where $\varepsilon_{F 0}=\pi \hbar^{2} n_{0} / m_{e}$ is the Fermi energy in the absence of irradiation. Substituting Eq. (36) into Eq. (39) and keeping in mind that the broadening $\Gamma_{s}$ is assumed to be very small, the resistivity of $2 \mathrm{D}$ system, $\rho=1 / \sigma$, can be written as a sum,

$$
\rho=\rho_{2 \mathrm{D}}+\rho_{\mathrm{Q}},
$$

where the first term describes the resistivity of $2 \mathrm{D}$ system arisen from the usual potential scattering of conduction electrons, whereas the second term,

$$
\rho_{\mathrm{Q}}=\frac{2 n_{s}}{\pi n_{e}}\left(\frac{h}{e^{2}}\right) \frac{\left(\Gamma_{s} / 2\right)^{2}}{\left(\varepsilon_{F}-\varepsilon_{s}\right)^{2}+\left(\Gamma_{s} / 2\right)^{2}},
$$

is of purely quantum nature and describes the scattering of conduction electrons trough the light-induced quasistationary bound states (the Breit-Wigner resonant scattering), where $h / e^{2}$ is the resistivity quantum. It follows from (43) that the quantum resistivity depends resonantly on the Fermi energy $\varepsilon_{F}$ with the resonance at $\varepsilon_{F}=$ $\varepsilon_{s}$ and the resonant amplitude is $\bar{\rho}_{\mathrm{Q}}=\left(2 n_{s} / \pi n_{e}\right)\left(h / e^{2}\right)$.

In the present analysis of electron transport, we assumed the most general types of the scattering potential $U(\mathbf{r})$ and $2 \mathrm{D}$ electron system. To proceed, we have to make some approximations. Let us restrict the following analysis by the case of short-range scatterers which are conventionally modeled in 2D systems by the deltapotential (see, e.g., Ref. 53). This corresponds, particularly, to a semiconductor quantum well doped by neutral atoms. Then we can apply Eqs. (17)-(18) to describe the energy of the quasi-stationary bound electron state, $\varepsilon_{s}$, and its broadening, $\Gamma_{s}$. Next, let us consider the 2D electron system in GaAs-based quantum well, where conduction electrons fill only the ground subband (the electron density is $\left.n_{0}=5 \cdot 10^{11} \mathrm{~cm}^{-2}\right)$ and the electron effective mass is $m_{e} \approx 0.067 m_{0}\left(m_{0}\right.$ is the electron mass in vacuum). In such modern quantum wells, the electron mobility is $\mu=|e| \tau_{e} / m_{e} \sim 10^{6}-10^{7} \mathrm{~cm}^{2} / \mathrm{V} \cdot \mathrm{s}$. Therefore, the condition (9) can be satisfied near the high-frequency boarder of the microwave range. For instance, we have $\omega \tau_{e} \sim 10$ for the field frequencies around $\nu=\omega / 2 \pi=100 \mathrm{GHz}$. Therefore, the photon energy of the dressing field can be chosen as $\hbar \omega=1 \mathrm{meV}$.

The dependence of the bound state energy, $\varepsilon_{s}$, on the irradiation intensity, $I=c E_{0}^{2} / 4 \pi$, is plotted in Fig. 2a, where the vertical arrow marks the resonant point $\left(\varepsilon_{s}=\right.$ $\left.\varepsilon_{F}\right)$. Far from the resonant point $\left(\left|\varepsilon_{s}-\varepsilon_{F}\right| \gg \Gamma_{s}\right)$, the resonant term (43) can be neglected and the total resistivity (42) is $\rho \approx \rho_{2 \mathrm{D}}$. On the left of the resonant point, the bound states are empty $\left(f_{s} \approx 0\right)$ and this resistivity
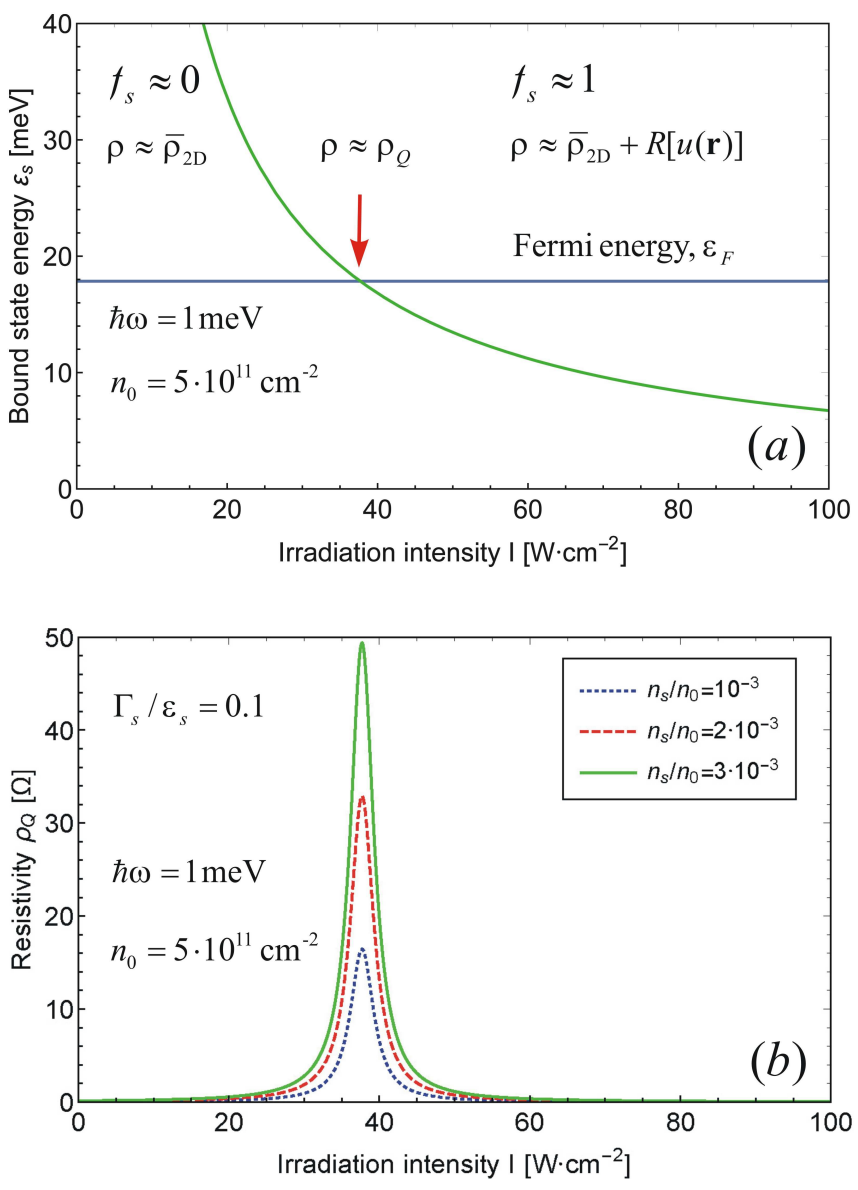

FIG. 2: Electronic characteristics of a GaAs-based quantum well filled by conduction electrons with the density $n_{0}=5 \cdot 10^{11} \mathrm{~cm}^{-2}$ and irradiated by a circularly polarized electromagnetic wave with the intensity $I$ and the photon energy $\hbar \omega=1 \mathrm{meV}$ : (a) Dependence of the bound state energy, $\varepsilon_{s}$, on the irradiation intensity, $I$, where the horizontal blue line marks the Fermi energy, $\varepsilon_{F}$, and the red vertical arrow marks the resonant point, $\varepsilon_{s}=\varepsilon_{F}$; (b) Dependence of the quantum resistivity, $\rho_{Q}$, on the irradiation intensity, $I$, for the energy broadening $\Gamma_{s}=0.1 \varepsilon_{s}$ and different scatterer densities, $n_{s}$.

reads $\rho_{2 \mathrm{D}}=\bar{\rho}_{2 \mathrm{D}}$, where

$$
\bar{\rho}_{2 \mathrm{D}}=\left(\frac{\rho_{0}}{2 \pi}\right) \int_{-\pi}^{\pi}(1-\cos \theta) J_{0}^{2}\left[2 k_{F} r_{0} \sin (\theta / 2)\right] d \theta,
$$

$\rho_{0}$ is the resistivity of the considered $2 \mathrm{D}$ system in the absence of irradiation, and $k_{F}=\sqrt{2 \pi n_{e}}$ is the Fermi wave vector of conduction electrons. It should be noted that Eq. (44) exactly coincides with the equation derived and analyzed in Ref. 15 beyond the effect of light-induced quasi-stationary electron states. On the right of the resonant point $\left(f_{s} \approx 1\right)$, the bound states are filled by captured electrons and the resistivity is $\rho_{2 \mathrm{D}}=\bar{\rho}_{2 \mathrm{D}}+R[u(\mathbf{r})]$, where $R[u(\mathbf{r})]$ is the functional depending on the scattering potential produced by a captured electron, $u(\mathbf{r})$. Physically, the addition $R[u(\mathbf{r})]$ to the resistivity (44) arises from the fact that the scatterers filled with cap- 
tured electrons scatter conduction electrons more effective than the empty scatterers. It should be noted that the capture of conduction electrons by the light-induced bound states leads also to the decreasing of density of conduction electrons, $n_{e}<n_{0}$ [see Eqs. (40)-(41)]. However, we have $n_{e} \approx n_{0}$ under the condition $n_{s} \ll n_{e}$ and, therefore, the contribution of decreasing density of conduction electrons to increasing resistivity is very small. Near the resonant point, we have to take into account the resistivity term (43). For typical parameters of the modern GaAs-based quantum well (the density of conduction electrons is $n_{0} \approx 5 \cdot 10^{11} \mathrm{~cm}^{-2}$ and the electron mobility is $\left.\mu \approx 10^{7} \mathrm{~cm}^{2} / \mathrm{V} \cdot \mathrm{s}\right)$, the resistivity of the system in the absence of irradiation is $\rho_{0} \approx 1 \Omega$, whereas the resistivity quantum is $h / e^{2} \approx 26 \mathrm{k} \Omega$. Since $h / e^{2} \gg \rho_{0}$, the resonant term (43) is dominant near the resonant point in the broad range of scatterer density, $n_{s}$. Therefore, we have $\rho \approx \rho_{Q}$ there. The dependence of the quantum resistivity (43) on the irradiation intensity, $I$, is plotted in Fig. 2b for different scatterer densities, $n_{s}$. It should be noted that the resonance amplitude of the resistivity (43) is still large enough, $\bar{\rho}_{Q} \sim 10^{-1} \Omega$, even if the scatterer density is very small, $n_{s} \sim 10^{-5} n_{e}$. Therefore, the quantum correction to the resistivity (43) can be detected in the state-of-the-art measurements in the broad range of scatterer densities.

In the present study, we considered the bound electron states induced in 2D systems by a monochromatic electromagnetic wave. It should be noted that such states take place in $1 \mathrm{D}$ systems as well ${ }^{46}$. As to $3 \mathrm{D}$ systems, the bound states disappear. Physically, this follows from the fact that alternating electric field of the wave can localize an electron only along the direction of its oscillations ${ }^{46}$. Since there are no field oscillations along the direction of propagation of the electromagnetic wave, the discussed mechanism of the light-induced electron localization does not work in 3D systems.

\section{CONCLUSION}

Irradiation of a 2D electron system by a circularly polarized off-resonant electromagnetic wave induces the quasi-stationary electron states confined at repulsive scatterers and immersed into the continuum of states of conduction electrons. These quasi-stationary bound electron states result in the corrections to conductivity of the irradiated 2D system through the two main mechanisms: The capture of conduction electrons by the bound states and the scattering of conduction electrons through these states. As a consequence, the corrections to resistivity of two kinds appear. The first of them is the non-resonant addition to the resistivity arisen from the increasing of the scattering of conduction electrons by the scatterers containing captured electrons. The second is the resonant addition to resistivity of purely quantum nature, which arises from the resonant Breit-Wigner scattering of conduction electrons through the quasi-stationary bound states (the resonant peak of the resistivity takes place when the Fermi energy of conduction electrons coincides with the quasi-discrete energy level of the bound state). Within the model of short-range scatterers described by the repulsive delta-potentials, the resistivity of GaAsbased quantum well is studied in the broad ranges of irradiation intensity and scatterer density.

\section{Acknowledgments}

The reported study was funded by the Russian Science Foundation (project 20-12-00001).

\section{Appendix A: Energy and broadening of quasi-stationary electron states}

Let us consider the Schrödinger problem with the Hamiltonian (13),

$$
\hat{\mathcal{H}}_{0}=-\frac{\hbar^{2}}{2 m_{e} r}\left[\frac{\partial}{\partial r}\left(r \frac{\partial}{\partial r}\right)+\frac{1}{r} \frac{\partial^{2}}{\partial \varphi^{2}}\right]+\frac{u_{0} \delta\left(r-r_{0}\right)}{2 \pi r_{0}}
$$

where $\varphi$ is the azimuth angle in the 2D plane. The eigenfunction of the Hamiltonian, $\psi$, which corresponds to an electron confined in the area $0<r<r_{0}$, must be finite at $r=0$ and satisfy the condition $\left.\psi\right|_{r \rightarrow \infty} \propto e^{i k r}$, where $k=\sqrt{2 m_{e} \varepsilon} / \hbar$ is the electron wave vector and $\varepsilon$ is the electron energy. Therefore, the sought wave function can be written with using the Bessel functions as

$$
\psi=e^{i m \varphi}\left\{\begin{array}{cl}
A J_{m}(k r), & 0<r<r_{0} \\
C H_{m}(k r), & r>r_{0}
\end{array},\right.
$$

where $m=0, \pm 1, \pm 2, \ldots$ is the angular electron momentum, $J_{m}(z)$ is the Bessel function of the first kind, $H_{m}(z)=J_{m}(z)+i N_{m}(z)$ is the Bessel function of the third kind (the Hankel function of the first kind), $N_{m}(z)$ is the Bessel function of the second kind (the Neumann function), and $A, C$ are the constants. Substituting the wave function (A2) into the Schrödinger equation with the Hamiltonian (A1) and integrating it over $r$ near $r=r_{0}$, we arrive at the continuity condition for electron current density at the ring-shape delta-potential barrier,

$$
C H_{m}^{\prime}(z)-A\left[J_{m}^{\prime}(z)+\frac{m_{e} u_{0}}{\pi \hbar^{2} k r_{0}} J_{m}(z)\right]=0,
$$

where $z=k r_{0}$. As to the continuity condition for the electron wave function $\mathrm{A} 2$ at the barrier, it reads

$$
C H_{m}(z)-A J_{m}(z)=0 .
$$

The two homogeneous algebraic equations (A3) and A4 define the constants $A$ and $C$, whereas the secular equation arisen from them,

$$
H_{m}^{\prime}(z) J_{m}(z)-H_{m}(z)\left[J_{m}^{\prime}(z)+\frac{m_{e} u_{0}}{\pi \hbar^{2} k r_{0}} J_{m}(z)\right]=0
$$


defines the total electron energy, $\varepsilon$. To simplify Eq. A5, let us apply the known equalities, $J_{\nu}(z) N_{\nu-1}(z)-$ $J_{\nu+1}(z) N_{\nu}(z)=-2 / \pi z$ and $Z_{\nu}^{\prime}(z)=\left[Z_{\nu-1}(z)-\right.$ $\left.Z_{\nu+1}(z)\right] / 2$, where $Z_{\nu}(z)$ is any Bessel function (see, e.g., Ref. 54). Then Eq. (A5) can be rewritten in the compact form as

$$
H_{m}(z) J_{m}(z)=i \alpha,
$$

where $z=k r_{0}$ and $\alpha=2 \hbar^{2} / m_{e} u_{0}$.

Although the secular equation (A6) can be easily solved numerically, there is the important particular case of strong repulsive potential, $\alpha \ll 1$, when solution of this equation can be found analytically. Namely, let us seek roots of Eq. A6 as a power series, $z_{n m}=$ $\bar{z}_{n m}+\sum_{l=1}^{\infty} z_{n m}^{(l)} \alpha^{l}$, where the integer $n$ numerates the roots and $z_{n m}^{(l)}$ are the expansion coefficients. Substitut$\operatorname{ing} z=z_{n m}$ into Eq. (A6) and expanding the Bessel functions there into the Taylor series near $\alpha=0$, we arrive at the system of algebraic recurrence equations for the expansion coefficients. The solving of the system results in the sought energy spectrum of an electron confined inside the ring-shape delta-barrier, $\varepsilon=\varepsilon_{n m}-i \Gamma_{n m} / 2$, and the corresponding wave functions (A2), where the energy of quasi-discrete electron level is

$$
\varepsilon_{n m}=\frac{\hbar^{2} \bar{z}_{n m}^{2}}{2 m_{e} r_{0}^{2}}+\mathcal{O}(\alpha),
$$

the broadening of the energy level is

$$
\Gamma_{n m}=\frac{4 \varepsilon_{n m} \alpha^{2}}{N_{m}^{3}\left(\bar{z}_{n m}\right)\left[J_{m+1}\left(\bar{z}_{n m}\right)-J_{m-1}\left(\bar{z}_{n m}\right)\right]}+\mathcal{O}\left(\alpha^{3}\right),
$$

the wave functions $\mathrm{A} 2$ read

$$
\begin{aligned}
& \psi_{n m}=\frac{e^{i m \varphi}}{\sqrt{\pi} r_{0} J_{m+1}\left(\bar{z}_{n m}\right)}\left\{\begin{array}{cl}
J_{m}\left(\frac{\bar{z}_{n m} r}{r_{0}}\right), & 0<r \leq r_{0} \\
0, & r \geq r_{0}
\end{array}\right. \\
& +\mathcal{O}(\alpha) \text {, }
\end{aligned}
$$

$\bar{z}_{n m}$ is the $n$th zero of the $m$ th Bessel function of the first kind (i.e. $J_{m}\left(\bar{z}_{n m}\right)=0$ ), and $n=1,2,3, \ldots$ is the principal quantum number.

\section{Appendix B: Probabilities of electron transitions}

The interaction between the bound electron state, $|s\rangle=\varphi_{s}(r)$, and the states of free conduction electrons, $|\mathbf{k}\rangle=\sqrt{1 / S} e^{i \mathbf{k r}}$, arises from the electron tunneling through the potential barrier, $|s\rangle \rightarrow|\mathbf{k}\rangle$ (see Fig. 1). Since the tunneling is assumed to be weak, it can be described in the most general form by the tunnel Hamiltonian

$$
\hat{\mathcal{H}}_{T}=|s\rangle \varepsilon_{s}\left\langle s\left|+\sum_{\mathbf{k}}\right| \mathbf{k}\right\rangle \varepsilon_{k}\left\langle\mathbf { k } \left|+\sum_{\mathbf{k}}\left[|\mathbf{k}\rangle\left|T_{\mathbf{k}}\right|\langle s|+\text { H.c. }\right],\right.\right.
$$

where $T_{\mathbf{k}}=\left\langle\mathbf{k}\left|\hat{\mathcal{H}}_{T}\right| s\right\rangle$ is the tunnel matrix element of the Hamiltonian between the localized and delocalized electron states. As to the summation over electron states with wave vectors $\mathbf{k}=\left(k_{x}, k_{y}\right)=(k \cos \theta, k \sin \theta)$ in the $2 \mathrm{D}$ system of the area $S$, it is equal to the following integration:

$$
\sum_{\mathbf{k}} \rightarrow \frac{S}{(2 \pi)^{2}} \int_{0}^{\infty} k d k \int_{0}^{2 \pi} d \theta
$$

The wave function satisfying the Schrödinger equation with the Hamiltonian (B1) can be written as $|\psi\rangle=$ $a_{s}(t) e^{-i \varepsilon_{s} t / \hbar}|s\rangle+\sum_{\mathbf{k}} a_{\mathbf{k}}(t) e^{-i \varepsilon_{k} t / \hbar}|k\rangle$. It should be noted that a scatterer is assumed to be centered at $\mathbf{R}=0$, where $\mathbf{R}$ is the radius vector of the scatterer position. In the most general case of $\mathbf{R} \neq 0$, the amplitudes $a_{\mathbf{k}}$ should be multiplied with the phase factor $e^{i \mathbf{k R}}$. Substituting this wave function into the Schrödinger equation, $i \hbar \partial_{t}|\psi\rangle=\hat{\mathcal{H}}|\psi\rangle$, and taking into account the approximate orthogonality of the basic states, $\langle\mathbf{k} \mid s\rangle=0$, we arrive at the quantum dynamics equations for the expansion coefficients $a_{s}(t)$ and $a_{\mathbf{k}}(t)$,

$$
\begin{aligned}
i \hbar \dot{a}_{s}(t) & =e^{\left.i\left(\varepsilon_{s}-\varepsilon_{k}\right) t / \hbar\right)} \sum_{\mathbf{k}} T_{\mathbf{k}}^{*} a_{\mathbf{k}}(t), \\
i \hbar \dot{a}_{\mathbf{k}}(t) & =e^{\left.i\left(\varepsilon_{k}-\varepsilon_{s}\right) t / \hbar\right)} T_{\mathbf{k}} a_{s}(t) .
\end{aligned}
$$

Let an electron be in the bound state at the time $t=0$, i.e. $a_{s}(0)=1$ and $a_{\mathbf{k}}(0)=0$. Then the integration of Eq. (B4) results in

$$
a_{\mathbf{k}}(t)=-\frac{i T_{\mathbf{k}}}{\hbar} \int_{0}^{t} e^{i\left(\varepsilon_{k}-\varepsilon_{s}\right) t^{\prime} / \hbar} a_{s}\left(t^{\prime}\right) d t^{\prime} .
$$

Since the considered system is axially symmetrical, the matrix element $T_{\mathbf{k}}$ depends only on the electron energy, $\varepsilon_{k}=\hbar^{2} k^{2} / 2 m$ and, therefore, can be denoted as $T_{\mathbf{k}}=$ $T_{\varepsilon_{k}}$. Substituting Eq. (B5) into Eq. (B3), we arrive at the expression

$$
\dot{a}_{s}(t)=-\frac{1}{\hbar^{2}} \sum_{\mathbf{k}}\left|T_{\varepsilon_{k}}\right|^{2} \int_{0}^{t} e^{i\left(\varepsilon_{k}-\varepsilon_{s}\right) t^{\prime} / \hbar} a_{s}\left(t^{\prime}\right) d t^{\prime},
$$

which can be rewritten as

$$
\begin{aligned}
& \dot{a}_{s}(t)= \\
& -\frac{S m_{e}}{2 \pi \hbar^{4}} \int_{0}^{\infty} d \varepsilon_{k}\left|T_{\varepsilon_{k}}\right|^{2} \int_{0}^{t} e^{i\left(\varepsilon_{s}-\varepsilon_{k}\right)\left(t-t^{\prime}\right) / \hbar} a_{s}\left(t^{\prime}\right) d t^{\prime} .
\end{aligned}
$$

This is still an exact equation since we just replaced two differential equations (B3)-(B4) with one linear differential-integral equation ( $\overline{\mathrm{B} 6}$ ). Next we make the approximation. Namely, let us take into account that the tunneling between the states $|s\rangle$ and $|\mathbf{k}\rangle$ is very weak. Then the quantity $\left|T_{\varepsilon_{k}}\right|^{2}$ varies little around $\varepsilon_{k}=\varepsilon_{s}$ for which the time integral in Eq. (B6) is not negligible. Physically, this means that the condition (19) is assumed 
to be satisfied and, therefore, the energy of outgoing electron, $\varepsilon_{k}$, is near the energy of bound state, $\varepsilon_{s}$.

As a consequence, one can make the replacement $\left|T_{\varepsilon_{k}}\right| \rightarrow\left|T_{\varepsilon_{s}}\right|$ and replace the lower limit in the $\varepsilon_{k}$ integration with $-\infty$. Since this integration results in the delta function,

$$
\int_{-\infty}^{\infty} e^{i\left(\varepsilon_{s}-\varepsilon_{k}\right)\left(t-t^{\prime}\right) / \hbar} d \varepsilon_{k}=2 \pi \hbar \delta\left(t-t^{\prime}\right),
$$

Eq. (B6) takes the form

$$
\dot{a}_{s}(t)=-\frac{\Gamma_{s}}{2 \hbar} a_{s}(t)
$$

where

$$
\Gamma_{s}=\frac{S m_{e}}{\hbar^{2}}\left|T_{\varepsilon_{s}}\right|^{2}
$$

is the energy broadening of the bound electron state. It follows from Eq. (B8) that

$$
a_{s}(t)=e^{-\Gamma_{s} t / 2 \hbar} .
$$

Now, we can calculate the probabilities $W_{\mathbf{k} s}$ and $w_{\mathbf{k k}^{\prime}}$ which appear in the Boltzmann kinetic equation [see Eqs. (22) and (27)]. Substituting Eq. (B9) into Eq. (B5), the amplitude of emission of free electron with the wave vector $\mathbf{k}$ from the bound state during the time $t$ reads

$$
a_{\mathbf{k}}(t)=-T_{\varepsilon_{k}} \frac{e^{i\left(\varepsilon_{k}-\varepsilon_{s}\right) t / \hbar-\Gamma_{s} t / 2 \hbar}-1}{\varepsilon_{k}-\varepsilon_{s}+i \Gamma_{s} / 2} .
$$

Making the replacement $T_{\varepsilon_{k}} \rightarrow T_{\varepsilon_{s}}$, the total amplitude of electron transition from the state $|s\rangle$ to the state $|\mathbf{k}\rangle$ is

$$
a_{\mathbf{k}}(\infty)=\frac{T_{\varepsilon_{s}}}{\varepsilon_{k}-\varepsilon_{s}+i \Gamma_{s} / 2} .
$$

Taking into account the reversibility of electron transitions and using Eq. (B8), the total probability of capture of a conduction electron with the wave vector $\mathbf{k}$ by a scatterer, $W_{s \mathbf{k}}=W_{\mathbf{k} s}=\left|a_{\mathbf{k}}(\infty)\right|^{2}$, can be written as

$$
W_{s \mathbf{k}}=\frac{\hbar^{2} \Gamma_{s}}{S m_{e}\left[\left(\varepsilon_{k}-\varepsilon_{s}\right)^{2}+\left(\Gamma_{s} / 2\right)^{2}\right]} .
$$

To describe the scattering of conduction electrons in the most general form, let us add the scattering Hamiltonian,

$$
\hat{\mathcal{H}}_{S}=\sum_{\mathbf{k}^{\prime \prime}} \sum_{\mathbf{k}^{\prime \prime \prime} \neq \mathbf{k}^{\prime \prime}}\left|\mathbf{k}^{\prime \prime}\right\rangle\left\langle\mathbf{k}^{\prime \prime}\left|U\left(\mathbf{r}-\mathbf{r}_{0}[t]\right)\right| \mathbf{k}^{\prime \prime \prime}\right\rangle\left\langle\mathbf{k}^{\prime \prime \prime}\right|,
$$

to the tunnel Hamiltonian (B1), where $U\left(\mathbf{r}-\mathbf{r}_{0}[t]\right)$ is the oscillating potential (6). Then the probability of electron scattering per unit time between the states $\left|\mathbf{k}^{\prime}\right\rangle$ and $|\mathbf{k}\rangle$, which takes into account both tunnel transitions arisen from the Hamiltonian (B1) and the potential scattering induced by the Hamiltonian (B13), is defined in the lowest order of the conventional perturbation theory $\underline{51}$ as

$$
\begin{aligned}
w_{\mathbf{k k}^{\prime}} & =\frac{2 \pi}{\hbar} \mid \sum_{j=1}^{N_{s}}\left[\frac{\left\langle\mathbf{k}\left|\hat{\mathcal{H}}_{T}\right| s\right\rangle\left\langle s\left|\hat{\mathcal{H}}_{T}\right| \mathbf{k}^{\prime}\right\rangle}{\varepsilon_{k}-\varepsilon_{s}+i \Gamma_{s} / 2}+\left\langle\mathbf{k}\left|U_{0}(\mathbf{r})\right| \mathbf{k}^{\prime}\right\rangle\right] \\
& \times\left. e^{i\left(\mathbf{k}^{\prime}-\mathbf{k}\right) \mathbf{R}_{j}}\right|^{2} \delta\left(\varepsilon_{k^{\prime}}-\varepsilon_{k}\right)
\end{aligned}
$$

where the summation index $j$ numerates different scatterers, and $U_{0}(\mathbf{r})$ is the time-averaged oscillating potential (8). The first term in the square brackets of Eq. (B14) corresponds to the tunnel electron scattering through the quasi-discrete energy level $\varepsilon_{s}$. As to the second term, it describes the elastic scattering of conduction electrons by the oscillating potential (6) and reads

$$
\left\langle\mathbf{k}\left|U_{0}(\mathbf{r})\right| \mathbf{k}^{\prime}\right\rangle=\frac{\gamma_{\mathbf{k k}} \mathbf{k}^{\prime}}{S} U_{\mathbf{k k}^{\prime}},
$$

where $U_{\mathbf{k k}^{\prime}}=\left\langle e^{i \mathbf{k r}}|U(\mathbf{r})| e^{i \mathbf{k}^{\prime} \mathbf{r}}\right\rangle$ is the matrix element of the Born scattering for the initial potential $U(\mathbf{r})$, the Bessel-function factor,

$$
\gamma_{\mathbf{k k}^{\prime}}=J_{0}\left[2 k r_{0} \sin (\theta / 2)\right],
$$

arises from oscillations of the scattering potential (6), and $\theta=\widehat{\mathbf{k}, \mathbf{k}^{\prime}}$ is the angle between the wave vectors of incident and scattered electron waves. Taking into account the random arrangement of scatterers and using Eq. (B8), the summation over the index $j$ in Eq. (B14) results in

$$
\begin{aligned}
w_{\mathbf{k k}^{\prime}} & =\frac{2 \pi n_{s}}{S \hbar} \mid \gamma_{\mathbf{k k}^{\prime}} U_{\mathbf{k k}^{\prime}} \\
& +\left.\frac{\hbar^{2} \Gamma_{s}}{m_{e}\left(\varepsilon_{k}-\varepsilon_{s}+i \Gamma_{s} / 2\right)}\right|^{2} \delta\left(\varepsilon_{k^{\prime}}-\varepsilon_{k}\right) .
\end{aligned}
$$

It should be noted that the probabilities discussed above are derived in the rotating reference frame (5), where the scattering potential (6) oscillates. However, they have the same form in the laboratory reference frame, where the kinetic Boltzmann equation depending on these probabilities is written (see Section III). Generally, such an invariance follows from the fact that an unitary transformation of the Hamiltonian to the new reference frame does not change matrix elements of physical quantities derived with using the Hamiltonian. In the particular case of the considered system, this invariance can be proved by a direct calculation of the matrix element (B15) in the laboratory reference frame, where the oscillating potential (6) turns into the stationary potential $U(\mathbf{r})$ but the plane electron waves $e^{i \mathbf{k r}}$ and $e^{i \mathbf{k}^{\prime} \mathbf{r}}$ should be replaced with the corresponding Floquet functions [the eigenfunctions of the first term of the Hamiltonian (1)]. As expected, the calculation results in the same matrix element (B15). Details of the calculation can be found in Appendix of Ref. 15, where the potential 
scattering of conduction electrons in 2D systems driven by an oscillating field was analyzed within the Floquet theory beyond the effect of field-induced quasi-stationary electron states.
* Electronic address: Oleg.Kibis()nstu.ru

1 N. Goldman and J. Dalibard, Periodically Driven Quantum Systems: Effective Hamiltonians and Engineered Gauge Fields, Phys. Rev. X 4, 031027 (2014).

2 M. Bukov, L. D'Alessio, and A. Polkovnikov, Universal high-frequency behavior of periodically driven systems: From dynamical stabilization to Floquet engineering, Adv. Phys. 64, 139 (2015).

3 F. Casas, J. A. Oteo, and J. Ros, Floquet theory: exponential perturbative treatment, J. Phys. A 34, 3379 (2001).

4 A. Eckardt and E. Anisimovas, High-frequency approximation for periodically driven quantum systems from a Floquet-space perspective, New J. Phys. 17, 093039 (2015).

5 S. Rahav, I. Gilary, and S. Fishman, Effective Hamiltonians for Periodically Driven Systems, Phys. Rev. A 68, 013820 (2003).

6 M. Holthaus, Floquet engineering with quasienergy bands of periodically driven optical lattices, J. Phys. B 49, 013001 (2015).

7 D. N. Basov, R. D. Averitt and D. Hsieh, Towards properties on demand in quantum materials, Nat. Mater. 16, 1077 (2017).

8 M. Vogl, P. Laurell, A. D. Barr, and G. A. Fiete, Flow Equation Approach to Periodically Driven Quantum Systems, Phys. Rev. X 9, 021037 (2019).

9 M. Vogl, M. Rodriguez-Vega, and G. A. Fiete, Effective Floquet Hamiltonian in the low-frequency regime, Phys. Rev. B 101, 024303 (2020).

10 O. V. Kibis, Dissipationless electron transport in photondressed nanostructures, Phys. Rev. Lett. 107, 106802 (2011).

11 K. L. Koshelevev, V. Yu. Kachorovskii, and M. Titov, Resonant inverse Faraday effect in nanorings, Phys. Rev. B 92, 235426 (2015).

12 V. K. Kozin, I. V. Iorsh, O. V. Kibis, I. A. Shelykh, Quantum ring with the Rashba spin-orbit interaction in the regime of strong light-matter coupling, Phys. Rev. B 97, 155434 (2018).

13 V. K. Kozin, I. V. Iorsh, O. V. Kibis, I. A. Shelykh, Periodic array of quantum rings strongly coupled to circularly polarized light as a topological insulator, Phys. Rev. B 97, 035416 (2018).

14 C. M. Yin, N. Tang, S. Zhang, J. X. Duan, F. J. Xu, J. Song, F. H. Mei, X. Q. Wang, B. Shen, Y. H. Chen, J. L. $\mathrm{Yu}$, and $\mathrm{H}$. Ma, Observation of the photoinduced anomalous Hall effect in GaN-based heterostructures, Appl. Phys. Lett. 98, 122104 (2011).

15 S. Morina, O. V. Kibis, A. A. Pervishko, I. A. Shelykh, Transport properties of a two-dimensional electron gas dressed by light, Phys. Rev. B 91, 155312 (2015).

16 A. A. Pervishko, O. V. Kibis, S. Morina, I. A. Shelykh, Control of spin dynamics in a two-dimensional electron gas by electromagnetic dressing, Phys. Rev. B 92, 205403 (2015).

17 K. Dini, O. V. Kibis, I. A. Shelykh, Magnetic properties of a two-dimensional electron gas strongly coupled to light,
Phys. Rev. B 93, 235411 (2016).

18 H. K. Avetissian, G. F. Mkrtchian, Nonlinear response of the quantum Hall system to a strong electromagnetic radiation, Phys. Lett. A 380, 3924 (2016).

19 N. H. Lindner, G. Refael and V. Galitski, Floquet topological insulator in semiconductor quantum wells, Nat. Phys. 7, 490 (2011).

20 M. C. Rechtsman, J. M. Zeuner, Y. Plotnik, Y. Lumer, D. Podolsky, F. Dreisow, S. Nolte, M. Segev and A. Szameit, Photonic Floquet topological insulator, Nature 496, 196 (2013).

21 Y. H. Wang, H. Steinberg, P. Jarillo-Herrero, and N. Gedik, Observation of Floquet-Bloch states on the surface of a topological insulator, Science 342, 453 (2013).

22 L. E. F. Foa Torres, P. M. Perez-Piskunow, C. A. Balseiro, G. Usaj, Multiterminal Conductance of a Floquet Topological Insulator, Phys. Rev. Lett. 113, 266801 (2014).

23 G. Usaj, P. M. Perez-Piskunow, L. E. F. Foa Torres, C. A. Balseiro, Irradiated graphene as a tunable Floquet topological insulator, Phys. Rev. B 90, 115423 (2014).

24 H. Dehghani, T. Oka, and A. Mitra, Out-of-equilibrium electrons and the Hall conductance of a Floquet topological insulator, Phys. Rev. B 91, 155422 (2015).

25 H. L. Calvo, L. E. F. Foa Torres, P. M. Perez-Piskunow, C. A. Balseiro, G. Usaj, Floquet interface states in illuminated threedimensional topological insulators, Phys. Rev. B 91, 241404(R) (2015).

26 T. Morimoto and N. Nagaosa, Topological nature of nonlinear optical effects in solids, Sci. Adv. 2, e1501524 (2016).

27 T. Mikami, S. Kitamura, K. Yasuda, N. Tsuji, T. Oka, H. Aoki, Brillouin-Wigner theory for high-frequency expansion in periodically driven systems: Application to Floquet topological insulators, Phys. Rev. B 93, 144307 (2016).

28 O. Kyriienko, O. V. Kibis, I. A. Shelykh, Optically induced topological states on the surface of mercury telluride, Phys. Rev. B 99, 115411 (2019).

29 T. Oka, H. Aoki, Photovoltaic Hall effect in graphene, Phys. Rev. B 79, 081406 (2009).

${ }^{30}$ O. V. Kibis, Metal-insulator transition in graphene induced by circularly polarized photons, Phys. Rev. B 81, 165433 (2010).

31 S. V. Syzranov, Ya. I. Rodionov, K. I. Kugel, F. Nori, Strongly anisotropic Dirac quasiparticles in irradiated graphene, Phys. Rev. B 88, 241112 (2013).

32 M. M. Glazov and S. D. Ganichev, High frequency electric field induced nonlinear effects in graphene, Phys. Rep. 535, 101 (2014).

33 P. M. Perez-Piskunow, G. Usaj, C. A. Balseiro, L. E. F. Foa Torres, Floquet chiral edge states in graphene, Phys. Rev. B 89, 121401(R) (2014).

34 M. A. Sentef, M. Claassen, A. F. Kemper, B. Moritz, T. Oka, J. K. Freericks and T. P. Devereaux, Theory of Floquet band formation and local pseudospin textures in pump-probe photoemission of graphene, Nat. Commun. 6, 7047 (2015).

35 E. J. Sie, J. W. McIver, Y.-H. Lee, L. Fu, J. Kong and N. Gedik, Valley-selective optical Stark effect in monolayer 
$\mathrm{WS}_{2}$, Nat. Mater. 14, 290 (2015).

36 O. V. Kibis, S. Morina, K. Dini, I. A. Shelykh, Magnetoelectronic properties of graphene dressed by a highfrequency field, Phys. Rev. B 93, 115420 (2016).

37 O. V. Kibis, K. Dini, I. V. Iorsh, I. A. Shelykh, All-optical band engineering of gapped Dirac materials, Phys. Rev. B 95, 125401 (2017).

38 I. V. Iorsh, K. Dini, O. V. Kibis, I. A. Shelykh, Optically induced Lifshitz transition in bilayer graphene, Phys. Rev. B 96, 155432 (2017).

39 S. A. Sato, J. W. McIver, M. Nuske, P. Tang, G. Jotzu, B. Schulte, H. Hubener, U. De Giovannini, L. Mathey, M. A. Sentef, A. Cavalleri, and A. Rubio, Microscopic theory for the light-induced anomalous Hall effect in graphene, Phys. Rev. B 99, 214302 (2019).

${ }^{40}$ P. Seifert, F. Sigger, J. Kiemle, K. Watanabe, T. Taniguchi, C. Kastl, U. Wurstbauer, and A. Holleitner, In-plane anisotropy of the photon-helicity induced linear Hall effect in few-layer $\mathrm{WTe}_{2}$, Phys. Rev. B. 99, 161403(R) (2019).

41 A. Iurov, G. Gumbs, D. H. Huang, Peculiar electronic states, symmetries, and Berry phases in irradiated alphaT(3)materials, Phys. Rev. B 99, 205135 (2019).

42 A. Iurov, L. Zhemchuzhna, D. Dahal, G. Gumbs, and D. Huang, Quantum-statistical theory for laser-tuned transport and optical conductivities of dressed electrons in alpha-T(3)materials, Phys. Rev. B 101, 035129 (2020).

43 J. W. McIver, B. Schulte, F.-U. Stein, T. Matsuyama, G.
Jotzu, G. Meier and A. Cavalleri, Light-induced anomalous Hall effect in graphene, Nat. Phys. 16, 38 (2020).

44 P. L. Kapitza, Dynamic stability of a pendulum when its point of suspension vibrates, Soviet Phys. JETP 21, 588 (1951).

45 E. van Duijn, M. Gavrila, and H. G. Muller, Multiply Charged Negative Ions of Hydrogen Induced by Superintense Laser Fields, Phys. Rev. Lett. 77, 3759 (1996).

46 O. V. Kibis, Electron pairing in nanostructures driven by an oscillating field, Phys. Rev. B. 99, 235416 (2019).

47 H. A. Kramers, Collected Scientific Papers (NorthHolland, Amsterdam, 1952).

48 W. C. Henneberger, Perturbation Method for Atoms in Intense Light Beams, Phys. Rev. Lett. 21, 838 (1968).

49 L. D. Landau and E. M. Lifshitz, The classical theory of fields (Butterworth-Heinemann, Oxford, 2000).

50 J. M. Ziman, Principles of the theory of solids (University Press, Cambridge, 1964).

51 L. D. Landau and E. M. Lifshitz, Quantum mechanics: Non-relativistic theory (Pergamon Press, Oxford, 1965).

${ }^{52}$ U. Fano, Effects of configuration interaction on intensities and phase shifts, Phys. Rev. 124, 1866 (1961).

53 T. Ando, A. B. Fowler, and F. Stern, Electronic properties of two-dimensional systems, Rev. Mod. Phys. 54, 437 (1982).

54 I. S. Gradstein and I. H. Ryzhik, Table of Series, Products and Integrals (Academic Press, New York, 2007). 